\title{
The Inhibitory Effect of Prunioside A Acyl Derivatives on NO Production in RAW 264.7 Cell
}

\author{
Woo-Yiel Lee, Byung Hee Kim, Young Hang Lee, Han Gil Choi, ${ }^{\dagger}$ Byung Hun Jeon, ${ }^{\ddagger}$ Seon Il Jang $\$$ \\ Young-Jun Kim," Hun Taeg Chung, ${ }^{,}$Young Soon Kim, and Kyu-Yun Chai"
}

\author{
Department of Bionanochemistry and Basic Sciences Research Institute, \\ Wonkwang Linversity, Iksan, Chonbuk 570-749, Korea \\ ${ }^{\dagger}$ Department of Biological Science, Honkwang Lniversity, Iksan, Chonbuk 570-749, Korea \\ ${ }^{\ddagger}$ Department of Pathologv. College of Oriental Medicine, Wonkwang Lniversity, Iksan, Chonbuk 570-749, Korea \\ ${ }^{\$}$ Department of Skin \& Beauty, Seojeong College, Iongju $+82-860$, Korea \\ ${ }^{~ " D e p a r t m e n t ~ o f ~ I m m u t h o l o g v, ~ I n s t i t u t e ~ o f ~ I m m t e c ~ K o r e a, ~ I k s a n, ~ C h o n b u k ~} 570-749$, Korea \\ १i fedicinal Resources Research Center of Honkwang Lniversity, Iksan, Chonbuk 570-749, Korea \\ Received July 21, 2004
}

\begin{abstract}
Pnunioside A is a unique. highly oxidized monoterpene glycoside isolated from the methanol extract of Spiraec primifolia var. Simpliciffora's root. The ester derivatives were synthesized from the hydrolyzed compounds of prunioside A by $\beta$-glucosidase. The derivatives showed suppressive effects on the generation of nitric oxide in murine machrophage-like RAW 264.7 cells stimulated by lipopolysaccharide and interferon- $\gamma$.
\end{abstract}

Key Words : Spiraea prunifolia, Prunioside A. Nitric oxide. NO production, Inlubitory effects

\section{Introduction}

Nitric oxide (NO). a small molecule produced in mammalian cells. has been known to participate in diverse physiological processes including platelet inhibition. blood pressure homeostasis. neurotransmission. immune responses. and inflammation. ${ }^{1.2}$ This molecule is produced by nitric oxide synthases (NOS) from L-arginine in certain cells. NO synthases can be classed into two types. constitutive nitric oxide sy'nthase (cNOS) and inducible nitric oxide sy'nthase (iNOS). The enzyme in macrophages is induced by cytokines such as lipopolysaccharide (LPS). interferon- $\gamma$ (IFN- $\gamma$ ). interlukin-2 (IL-2), and tumor necrosis factor- $\alpha$ (TNF- $\alpha$ ). NO synthesized by the inducible enzyme has showed cytotoxic effects on microbes. tumor cells. macrophages. and lymphocytes. However the increased NO production has been known to cause TNF-induced hypotension and various inflammatory diseases ${ }^{3-5}$ Therefore. many studies have been carried out to obtain a potential therapeutic agent from various natural substances which has the inhibitory effect on NO production by iNOS.

Recently: the methanol extract from Spircea prunifolia var. simpliciffora root has been reported to suppress the generation of NO in polymyristic acetate stimulated RAW 264.7 cells. Spircea prinifolia var. simplicifora (Rosaceae). a deciduous and latifoliate slurub. is found in the most parts of Korea and it's roots has been traditionally used for the treatment of fever and emetic conditions. ${ }^{7 .}$ We have found the acetylated derivative of a secondary metabolite pnunioside A from the roots of the plant showing the inhibitory effect on iNOS. In this study we have synthesized the

\footnotetext{
${ }^{*}$ Corresponding Author. Tel: $-82-63-850-6230$ : Fax: $-82-63-841$ -
} 4893: e-117ail: gellyonia wonkwang.ac.kr various ester derivatives of the hydrolyzed compounds. prepared from prunioside A by $\beta$-glucosidase, and analyzed their inhibitory effect on NO production in murine macrophage-like RAW 264.7 cells stimulated with INF- $\gamma$ and lipopoly saccharide (LPS).

\section{Experimental Section}

General experimental procedures. ESIMS data were obtained on a MicroMass Quatro LC with electro-spray ionization. NMR spectra were recorded in either acetone- $d s$ or $\mathrm{CDCl}_{3}$ using a JEOL Eclipse- $500 \mathrm{MHz}$ spectrometer $(500$ $\mathrm{MHz}$ for ${ }^{1} \mathrm{H}$ and $125 \mathrm{MHz}$ for ${ }^{13} \mathrm{C}$ ). and chemical shifts were referenced relative to the corresponding residual solvent signals (acetone- $d_{6}: \delta 2.04 / 29.9 . \mathrm{CDCl}_{3}: \delta 7.24 / 77.0$ ). HPLC separations were performed on a Symmetry Prep $\mathrm{C}_{18}$ columnn $(1.9 \times 30 \mathrm{~cm}: 7-\mu \mathrm{m}$ particle size: flow rate of $4 \mathrm{~mL} / \mathrm{min})$. Compounds were detected by measuring UV absorption at 210 and $254 \mathrm{~nm}$ on a Hewlett-Packard HP 8453 spectrophotometer.

Extraction and isolation of prunioside $A$ (1). The roots of S. prunifolia were collected from Iksan City. Chonbuk Province. Korea in May 2002 and dried as previously

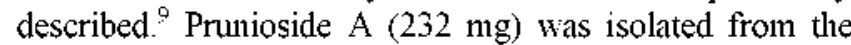
methanol extract of the air-dried roots $(1 \mathrm{~kg})$ of $S$ prunifolia according to the previous method. ${ }^{\text {(c) }}$

Enzymatic hydrolysis of prunioside $A$ (1) with $\beta$ glucosidase. The enzymatic reaction was carrried out by incubating a solution of compound $\mathbf{l}(92 \mathrm{mg})$ in $\mathrm{H}_{2} \mathrm{O}$ (10 $\mathrm{mL}$ ) with $\beta$-glucosidase ( $46 \mathrm{mg}$ ) from yeast ( $\mathrm{G} 8889$. Sigma Chemical Co.. St. Louis. MO) at $37^{\circ} \mathrm{C}$ for $12 \mathrm{~h}$. The reaction mixture was extracted with EtOAc $(3 \times 10 \mathrm{~mL})$. The dried organic residue was applied to reversed-phase HPLC to afford 3-(4-Hydroxy-phenyl)-acrylic acid 1-[4-(2-hydroxy- 
ethylidene)-5-oxo-tetrahydro-furan-2-yl]-2-methyl-allyl ester (compound 2. $40 \mathrm{mg}$ ) (Scheme 1): ${ }^{1} \mathrm{H}$ NMR $(500 \mathrm{MHz}$. acetone- $\left.d_{6}\right): \delta 1.82(3 \mathrm{H}, s) .2 .75(1 \mathrm{H}, m) .3 .08(\mathrm{lH} . m) .4 .58$ $(1 \mathrm{H}, m) .4 .64(1 \mathrm{H}, m) .4 .91(\mathrm{lH}, d d d . J=8.3 .5 .5,5.5), 5.04$ $(1 \mathrm{H}, b r s) .5 .10(\mathrm{lH}$. br s) $5.40(1 \mathrm{H}, d, J=5.5) .6 .32(\mathrm{lH}$. m). $6.38(\mathrm{lH} . d . J=16.0 \mathrm{~Hz}),, 6.89(2 \mathrm{H} . d . J=8.7 \mathrm{~Hz}) .7 .56$ $(2 \mathrm{H}, d . J=8.7 \mathrm{~Hz}), 7.62(1 \mathrm{H}, d . J=16.0 \mathrm{~Hz}) ;{ }^{13} \mathrm{C}$ NMR: $\delta$ $18.5,30.9,58.1 .76 .7 .77 .4,114.1,114.4,115.9 .124 .3$. $126.0,130.3,140.5,143.0,145.5,160.0,165.6 .168 .9$; ESIMS: $m: 3+5\left(100 \% . \mathrm{M}+\mathrm{H}^{-}\right)$.

Benzoic acid 4-[2-(2-methyl-1-\{5-0xo-4-[2-(2-vinyl-but2-enoyloxy)-ethylidene]-tetrahydro-furan-2-yl\}-allyloxycarbonyl)-vinyl]-phenyl ester (3). A solution of 2 (5 $\mathrm{mg}$. $\left.1.46 \times 10^{-5} \mathrm{~mol}\right)$. 4-N,N-dimetlly laninopyridine $(0.5 \mathrm{mg})$. and benzoyl chloride ( $40 \mu$ L. $2.85 \times 10^{-7} \mathrm{~mol}$ ) in acetonitrile ( $2 \mathrm{~mL}$ ) was stirred for $30 \mathrm{~min}$ at room temperature. The ester compound 3 (5.5 $\mathrm{mg}$, yield $68 \%$ ) was obtained by the separation procedure described above: ${ }^{3} \mathrm{H}$ NMR $(500 \mathrm{MHz}$. acetone- $\left.d_{6}\right): \delta 1.86(3 \mathrm{H}, s) .2 .82-2.89(1 \mathrm{H}, m), 3.17(1 \mathrm{H}, \mathrm{m})$. $5.02(\mathrm{lH} . d d d . J=5.1 .5 .1,8.3) .5 .07(1 \mathrm{H}, s) .5 .14(1 \mathrm{H}, s)$. $5.36-5.49(2 \mathrm{H} . m), 5.48(\mathrm{lH} . d, J=4.6) .6 .41(1 \mathrm{H}, m) .6 .63$ $(1 \mathrm{H}, d, J=16.1), 7.33(2 \mathrm{H}, d . J=8.7), 7.46(2 \mathrm{H}, t, J=8.3)$. $7.59(2 \mathrm{H}, t, J=7.8) .7 .71(2 \mathrm{H}, m), 7.8 \mathrm{I}(2 \mathrm{H} . d, J=8.7), 7.95$ $(2 \mathrm{H} . d . J=8.3), 8.17(2 \mathrm{H}, d, J=7.3):{ }^{13} \mathrm{C} \mathrm{NMR}: \delta 17.8,24.9,63$. $80.8,85.8 .107 .6,118.1,121.3,126.6,128.4 .128 .5 .129 .7$. $130.1,130.5,130.6,131.7,132.8,133.7,135.6,136.2$. 143.3, 147.9. 152.3. 164, 165.5. 165.7. 167: ESI-MS: $m z$ $575\left(100 \% . \mathrm{M}+\mathrm{Na}^{-}\right)$.

Cyclopentanecarboxylic acid 4-(2-\{1-[4-(2-cyclopentanecarbony-loxyethylidene)-5-0xo-tetrahydro-furan-2-yl]-2methylallyloxy-carbonyl\}-vinyl)-phenyl ester (4). A solution of $2\left(5 \mathrm{mg}, 1.46 \times 10^{-5} \mathrm{~mol}\right)$. 4-N, $N$-dimethylaninopyridine $(0.5 \mathrm{mg})$, and cyclopentanecarbonyl chloride (40 $\left.\mu \mathrm{L} .3 .03 \times 10^{-7} \mathrm{~mol}\right)$ in acetonitrile $(2 \mathrm{~nL})$ was allowed for the esterification at room temperature for $30 \mathrm{~min}$. The HPLC separation followed by the solvent extraction gave $5.6 \mathrm{mg}$ of compound 4 (yield $72 \%)$; ${ }^{1} \mathrm{H}$ NMR $\left(500 \mathrm{MHz}\right.$, acetone- $\left.d_{6}\right)$ : $\delta$ $1.5 \mathrm{l}-1.98(18 \mathrm{H}, m), 1.83(3 \mathrm{H}, s), 2.84(1 \mathrm{H}, m) .3 .06(\mathrm{lH} . m)$. $4.98(\mathrm{lH} . d d d, J=4.6 .4 .6,8.7) .5 .09-5.16(2 \mathrm{H}, m), 5.05$ $(1 \mathrm{H}, s), 5.11(1 \mathrm{H}, s) .5 .45(\mathrm{lH} . d, J=4.6), 6.23(1 \mathrm{H}, m) .6 .60$ $(1 \mathrm{H}, d . J=16.0) .7 .20(2 \mathrm{H}, d . J=8.7), 7.71(1 \mathrm{H} . d . J=16.1)$. $7.77(2 \mathrm{H}, d .8 .7) ;{ }^{13} \mathrm{C}$ NMR: $\delta 17.8 .22 .8 .23 .1,24.9 .25 .4$. $25.4,40.8 .41 .3,63.7 .80 .8,85.8$. 107.6. 118.1. 121.3. 126.6 . $131.7,135.6 .136 .2 .143 .3,147.9,152.3 .165 .5 .165 .7 .173$. 176; ESI-MS: $m z 559\left(100 \% . \mathrm{M}+\mathrm{Na}^{+}\right)$.

3-Methyl-but-2-enoic acid +-[2-(2-methy]-1-\{4-[2-(3methyl-but-2-enoyloxy)-ethylidene]-5-oxo-tetrahydro-furan2-yl)-allyloxycarbonyl)-vinyl]-phenyl ester (5). To a solution of 2 (5 mg. $\left.1.46 \times 10^{-5} \mathrm{~mol}\right)$. 4-N,N-dimethylaminopyridine $(0.5 \mathrm{mg})$ in acetonitrile ( $2 \mathrm{~mL}$ ) was added dimetlyy lacryloyl chloride ( $40 \mu \mathrm{L} .3 .38 \times 10^{-7} \mathrm{~mol}$ ). The reaction mixture was stirred for $30 \mathrm{~min}$ at room temperature. The dried organic residue obtained from the solvent extraction with $\mathrm{EtOAC}$ and $\mathrm{H}_{2} \mathrm{O}$ was subjected to reversed-phase HPLC to afford $5.6 \mathrm{mg}$ (yield $76 \%$ ) of compound 5: ' $\mathrm{H}$ NMR $(500 \mathrm{MHz}$. acetone$\left.d_{6}\right): \delta 1.73(3 \mathrm{H} . s), 1.84(3 \mathrm{H} . s), 2.00(3 \mathrm{H}, s), 2.20(3 \mathrm{H}, s)$. 2.78-2.84 (lH. $m$ ). $3.00(2 \mathrm{H}, s), 3.12$ (lH. $m), 4.78$ (lH. $s$ ).
$4.83(1 \mathrm{H}, s) .4 .96(1 \mathrm{H}, d d d, J=4.6,4.6 .8 .7), 5.06(\mathrm{lH} . s)$ $5.11(\mathrm{lH} . s), 5.16(2 \mathrm{H}, m), 5.44(1 \mathrm{H}, d . J=4.6), 5.93(\mathrm{lH} . s)$, $6.23(\mathrm{lH} . m) .6 .57$ (1H. $d, J=16.1) .7 .19(2 \mathrm{H} . d, J=8.7)$, $7.68(1 \mathrm{H}, d, J=16.1), 7.75(2 \mathrm{H}, d, 8.3):{ }^{13} \mathrm{C}$ NMR: $\delta 17.8$. 18.7. 19.1. 24.7, 24.7, 24.9, 63.5. 80.8. 85.8, 107.6. 113.1, $115.6,118.1,121.3 .126 .6 .131 .7,135.6,136.2 .143 .3$, 147.6, 147.9, 151.9. 152.3. 165.5, 165.7. 168, 171: ESI-MS: $m z 531\left(100 \%, \mathrm{M}+\mathrm{Na}^{+}\right)$.

3-(4-Yryloxy-phenyl)-acrylic acid 1-[4-(2-butyryloxyethylidene)-5-oxo-tetrahydro-furan-2-yl]-2-methyl-allyl ester (6). A solution of $2\left(5 \mathrm{mg}, 1.46 \times 10^{-5} \mathrm{~mol}\right), 4-\mathrm{N}, \mathrm{N}$ dimetlyy lamino-pyridine $(0.5 \mathrm{mg})$, and butyryl chloride (4 $\left.\mu \mathrm{L}, 3.75 \times 10^{-7} \mathrm{~mol}\right) \mathrm{in}$ acetonitrile $(2 \mathrm{~mL})$ was stirred at room temperature for $30 \mathrm{~min}$. The solvent was evaporated under $\mathrm{N}_{2}$. The resulting residue was purified as described above to afford $5.1 \mathrm{mg}$ (yield $73 \%$ ) of compound $6 ;{ }^{3} \mathrm{H}$ NMR $\left(500 \mathrm{MHz}\right.$, acetone- $\left.d_{6}\right): \delta 0.88(3 \mathrm{H} . t . J=7.4) .1 .00$ (3H. $t, J=7.3) .1 .56(2 \mathrm{H} . m) .1 .73(2 \mathrm{H} . m) .1 .84(3 \mathrm{H} . s)$, $2.20(2 \mathrm{H}, t, J=7.3) .2 .57(2 \mathrm{H} . t . J=7.4) .2 .80(1 \mathrm{H}, m) .3 .15$ (lH. $m) .4 .98(\mathrm{lH}, d d d . J=5.0,5.0 .8 .3) .5 .05-5.15(2 \mathrm{H} . m)$, $5.05(\mathrm{lH} . s), 5.1 \mathrm{l}(\mathrm{lH}, s), 5.45(\mathrm{lH}, d, J=4.6), 6.23(\mathrm{lH}, m)$, $6.59(\mathrm{lH}, d . J=16.1), 7.20(2 \mathrm{H} . d . J=8.7), 7.70(\mathrm{lH} . d . J=$ 16.0). $7.75\left(2 \mathrm{H}\right.$. d. 8.7); ${ }^{13} \mathrm{C}$ NMR: $\delta$ 13.4. 13.7. 17.8, 18.2 . 18.2. 24.9. 35.4. 35.9, 63.4, 80.8, 85.8, 107.6, 118.1. 121.3, 126.6, 131.7, 135.6. 136.2. 143.3, 147.9. 152.3. 165.5, 165.7, 169, 172; ESI-MS: $m z 507\left(100 \% . \mathrm{M}+\mathrm{Na}^{-}\right)$.

Benzoic acid 4-(2-\{1-[4-(2-hydroxy-ethylidene)-5-ox0tetrahydro-furan-2-yl]-2-methyl-allyloxycarbonyl\}-vinyl)phenyl ester (7). Compound $2\left(5 \mathrm{mg}\right.$. $1.46 \times 10^{-5} \mathrm{~mol}$ ) was stirred with $4-N N$-dimethylaminopyridine $(0.5 \mathrm{mg})$ and benzoyl chloride $\left(2 \mu \mathrm{L}, 1.42 \times 10^{-7} \mathrm{~mol}\right)$ in acetonitrile $(2$ $\mathrm{mL}$ ) for $15 \mathrm{~min}$ at room temperature. Compound 7 (4.3 $\mathrm{mg}$. yield $66 \%$ ) was obtaind by the separation procedures described above; ${ }^{1} \mathrm{H}$ NMR $\left(500 \mathrm{MHz}\right.$, acetone- $\left.d_{6}\right): \delta 1.84$ (3H. s), 2.76-2.83 (lH, m), 3.09 (1H. $m), 4.61(2 \mathrm{H}, m), 4.92$ $(\mathrm{lH} . d d d, J=5.5 .5 .5,8.3), 5.06(\mathrm{lH}, s), 5.12(\mathrm{lH}, s), 5.43$ (lH. $d . J=5.1) .6 .32(\mathrm{lH}, m), 6.60(\mathrm{lH}, d . J=16.1), 7.37$ $(2 \mathrm{H} . d . J=8.7) .7 .59(2 \mathrm{H} . t . J=8.3), 7.72(2 \mathrm{H} . t . J=8.3)$, $7.82(2 \mathrm{H} . d, J=8.7) .8 .17(2 \mathrm{H}, d, J=7.4) ;{ }^{33} \mathrm{C}$ NMR: $\delta 17.8$, 24.9. 59.7. 80.8. 85.8. 107.6, 118.1. 121.3, 126.6. 128.5, 130.1. 130.6, 131.7. 133.7, 135.6. 136.2, 143.3. 147.9. 152.3. 164. 165.5, 165.7: ESI-MS: $m z 471\left(100 \%, \mathrm{M}+\mathrm{Na}^{+}\right)$.

Cyclopentanecarboxylic acid + -(2-\{1-[4-(2-hydroxyethylidene)-5-oxo-tetrahydro-furan-2-yl]-2-methyl-allyloxycarbonyl;-vinyl)-phenyl ester (8). Compound 8 (4.6 mg. yield $72 \%$ ) was prepared by allowing the reaction for $15 \mathrm{~min}$ from compound and cyclopentanecarbonyl chloride $(20 \mu \mathrm{L}$. $\left.1.51 \times 10^{-7} \mathrm{~mol}\right)$ using the acylation procedure described above: 'H NMR $(500 \mathrm{MHz}$, acetone- $d$ ) : $\delta 1.62-2.00(9 \mathrm{H}$. $m), 1.84(3 \mathrm{H}, s) .2 .78-2.83(\mathrm{lH}, m), 3.08(1 \mathrm{H}, m), 4.6 \mathrm{l}(2 \mathrm{H}$, $m), 4.91(\mathrm{lH} . d d d, J=5.1 .5 .1 .8 .7), 5.05(1 \mathrm{H} . s) .5 .11(\mathrm{lH}$, $s) .5 .4 \mathrm{l}(\mathrm{lH} . d, J=5.1), 6.32(\mathrm{lH} . m) .6 .55(\mathrm{lH} . d, J=\mathrm{I} 6.1)$, $7.18(2 \mathrm{H} . d . J=8.7), 7.67(\mathrm{lH}, d, J=16.1), 7.74(2 \mathrm{H}, d$, 8.7): ${ }^{13} \mathrm{C}$ NMR: $\delta 17.8,22.8$. 24.9. 25.4, 40.8. 59.7. 80.8, 85.8. 107.6, 118.1. 121.3, 126.6. 131.7. 135.6, 136.2. 143.3, $147.9,152.3 .165 .5 .165 .7,173$; ESI-MS: $m z 463(100 \%$. M $+\mathrm{Na}^{+}$). 

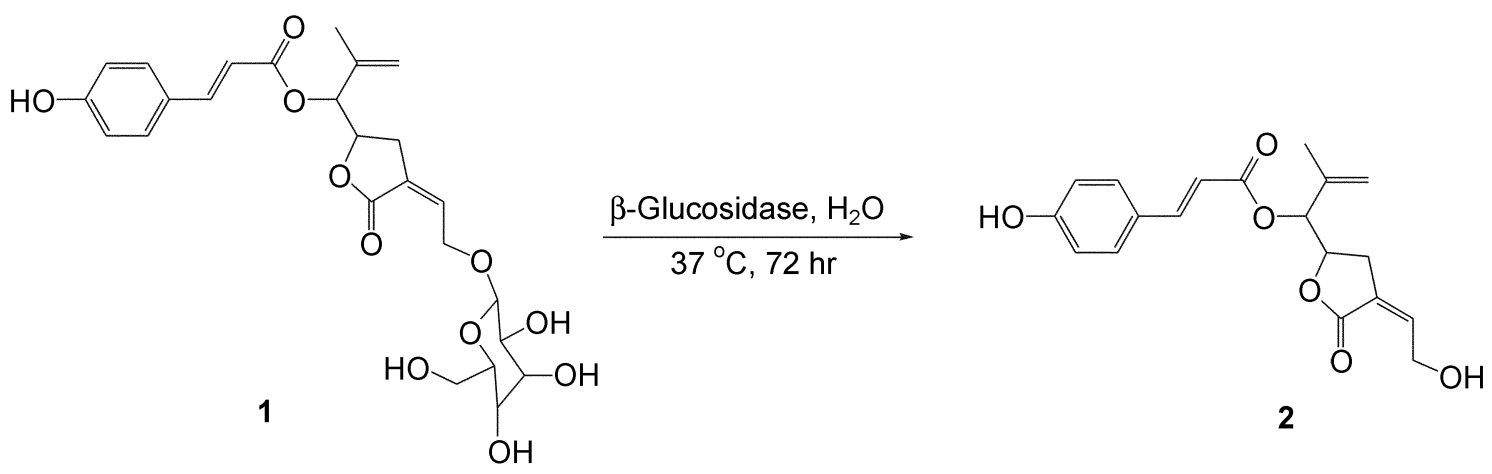

Scheme 1

3-Methyl-but-2-enoic acid 4-(2-\{1-|4-(2-hydroxy-ethylidene)-5-oxo-tetrahydro-furan-2-yl|-2-methyl-allyloxycarbonyl;-vinyl)-phenyl ester (9). Dimethylacryloyl chloride $\left(20 \mu \mathrm{l}, 1.69 \times 10^{-7} \mathrm{~mol}\right.$ ) was used to synthesize compound 9 (4.7 $\mathrm{mg}$, yield $76 \%$ ) from compound 2 ; ' $\mathrm{H} \mathrm{NMR} \mathrm{(500} \mathrm{MHz.}$ acetone- $\left.d_{6}\right): \delta 1.83\left(3 \mathrm{H}, s^{\prime}\right), 2.00(3 \mathrm{H}, s), 2.19(3 \mathrm{H}, s), 2.75-$ $2.81(1 \mathrm{H}, m), 3.06(1 \mathrm{H}, m), 4.61(2 \mathrm{H}, m), 4.92(1 \mathrm{H}, d d d, J=$ $5.5,5.5,8.3), 5.05(1 \mathrm{H}, s), 5.11(1 \mathrm{H}, s), 5.16(2 \mathrm{H}, m), 5.42$ $(1 \mathrm{H}, d, j=5.1), 5.92(1 \mathrm{H}, b H, s), 6.31(1 \mathrm{H}, m), 6.56(1 \mathrm{H}, d, J$ $=16.0), 7.19(2 \mathrm{H}, d, J=8.8), 7.68(1 \mathrm{H}, d, J=16.1), 7.75$ $(2 \mathrm{H}, d, 8.7)$; ${ }^{1.7} \mathrm{C}$ NMR: $\delta 17.8,18.7,24.7,24.9,59.7,80.8$, $85.8,107.6,113.1,118.1,121.3,126.6,131.7,135.6,136.2$, $143.3,147.9,151.9,152.3,165.5,165.7,168:$ ESI-MS: $m / z$ $449\left(100 \%, \mathrm{M}-\mathrm{Na}^{-}\right)$.

3-(4-Butyryloxy-phenyl)-acrylic acid 1-|4-(2-hydroxyethylidene)-5-oxo-tetrahydro-furan-2-yl|-2-methyl-allyl ester (10). Compound $10(4.6 \mathrm{mg}$. yield $77 \%)$ was obtained from butyryl chloride $\left(20 \mu \mathrm{l} ., 1.88 \times 10^{-7} \mathrm{~mol}\right)$ and compound $2 ;{ }^{1} \mathrm{H}$ NMR $\left(500 \mathrm{MH} 7\right.$, acetone- $\left.d_{6}\right) ; \delta 1.01\left(3 \mathrm{H}_{n} t\right.$. $J=7.4), 1.75(2 \mathrm{H}, m), 1.84(3 \mathrm{H}, s), 2.58(2 \mathrm{H}, t, J=7.4)$, $2.80(1 \mathrm{H}, m), 3.08(1 \mathrm{H}, m), 4.60(2 \mathrm{H}, m), 4.93(1 \mathrm{H} . d d d, J=$ $5.5,5.5,8.3), 5.05(1 \mathrm{H}, s), 5.11(1 \mathrm{H}, s), 5.42(1 \mathrm{H}, d . J=5.1)$, $6.32(1 \mathrm{H}, m), 6.58(1 \mathrm{H}, d . J=16.1), 7.20(2 \mathrm{H}, d . J=8.7)$. $7.71(1 \mathrm{H}, d . J=16.1), 7.76(2 \mathrm{H}, d .8 .7) ;{ }^{13} \mathrm{C}$ NMR: $\delta 13.4$, $17.8,18.2,24.9,35.4,59.7,80.8,85.8,107.6,118.1,121.3$, $126.6,131.7,135.6,136.2,143.3,147.9,152.3,165.5$, 165.7, 169; ESI-MS: $m / z 437\left(100 \%, \mathrm{M}+\mathrm{Na}^{-}\right)$.

Macrophage cell line culture. The murine macrophage cell line RAW264.7 was purchased from the American Jissue Culture Collection (Rockville, MD). The cells were maintained in complete RPMI 1640 medium supplemented with $10 \%$ heat inactivated fetal bovine serum, $1 \% \mathrm{~L}$ glutamine, $1 \%$ nonessential amino acids, $1 \%$ antibiotic/ antimycotic ( $100 \mathrm{U} / \mathrm{mL}$ of penicillin, $25 \mu \mathrm{g} / \mathrm{mL}$ of amphotericin $\mathrm{D}$, and $100 \mathrm{mg} / \mathrm{mL}$ of streptomycin). $1.5 \%$ sodium bicarbonate, and $1 \%$ minimal essential vitamins at $37{ }^{\circ} \mathrm{C}$ in a humidified $5 \% \mathrm{CO}_{2}$ atmosphere

Measurement of nitrite concentration. Experiments were undertaken on cells grown in the presence of various concentrations of test compounds dissolved in DMSO with LPS ( $1 \mu \mathrm{g} / \mathrm{mL})$ for $18 \mathrm{hr}$. The final concentration of DMSO in culture media was $0.1 \%$. Supernatants in cultured macrophages were collected and mixed with an equal volume of the Griess reagent ( $1 \%$ sulfanilamide, $0.1 \% N-(1-$ naphthyl)-ethylenediamine dihydrochloride in $2.5 \%$ phosphoric acid solution) and incubated for $10 \mathrm{~min}$ at room temperature. Nitrite concentration was determined by measuring the absorbance at $540 \mathrm{~nm}$ using an FI.ISA plate reader. The level of nitrite reflects nitric oxide synthesis. Sodium nitrite was used as a standard. The cell-free medium contained $5-8 \mu \mathrm{M}$ of nitrite, and this value was determined in each experiment and subtracted from the value obtained with cells.

\section{Results and Discussion}

NO, which is produced in a large amount by cytokines such as L.PS and IFN- $\gamma$ in macrphages and several immune cells, can be deleterious to the host and has been implicated in the pathogenesis of various inflammatory diseases. 1.5 Prunioside $A$, a unicjue terpene glycoside, has been recently identified from the methanol extract of S. primiofoliais roots showing a suppressive effect on NO production in murine macrophage-like RAW 264.7 cells stimulated with JFN- $\gamma$ plus I.PS. Though the terpene glycoside did not show any inhibitory effect on NO production in IFN- $\gamma$ and I.PSstimulated macrophage-like RAW 264.7 cells, its acetylated derivatives showed the inhibitory effect on the NO generation. ${ }^{9.10}$

The various ester derivatives were synthesized from the hydrolyzed prunioside A with $\beta$-glucosidase (Scheme 1) and their inhibitory effect on NO production ( $\left.\mathrm{IC}_{50}\right)$ of RAW 264.7 cells were determined (lable 1). Figure 1 showes the biological activities of ester derivatives (compounds 3-10) on NO production in the RAW 264.7 cells. All of them exhibited a clear suppressive effect on $\mathrm{NO}$ production in a dose-dependent manner in a range from $1 \mu \mathrm{g} / \mathrm{mL}$ to $50 \mu \mathrm{g} /$ $\mathrm{mL}$. Particularly, it is noticeable that compound $\mathbf{1 0}$ displays the most apparent inhibitory effect among the compounds examined (Fig. 1). RI-PCR (Reverse transcriptase-polymerase chain reaction) was conducted to determine whether the inhibition of $\mathrm{NO}$ production by compound $\mathbf{1 0}$ was owing to the interfering with the transcription of iNOS. The inhibitory effect on NO production by the compound $\mathbf{1 0}$ in IF $\mathrm{N}-\gamma$ and LPS-stimulated macrophage-like RAW 264.7 cells appeared to be correlated with the suppression of iNOS mRNA (data not shown).

In addition to the synthesis of various ester derivatives 
Table 1. Ithibitory effect of prunioside A derivatives on NO production

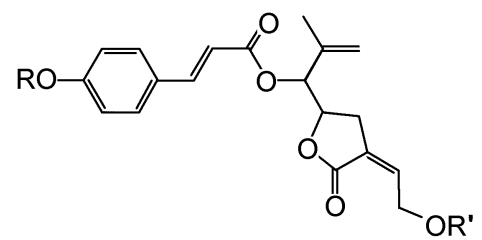

The ester derivatives of 2

\begin{tabular}{|c|c|c|c|c|c|}
\hline Entry & $\mathrm{R}$ & $\mathrm{R}^{\prime}$ & P'roduct & $\begin{array}{l}\text { Yield } \\
(\%)\end{array}$ & $\begin{array}{c}\mathrm{C}_{5 n} \\
\text { Inhibition }\end{array}$ \\
\hline 1 & & & 3 & 68 & $5.0 \mu \mathrm{g} / \mathrm{mL}$ \\
\hline 2 & & & 4 & 72 & $6.0 \mu \mathrm{g} / \mathrm{mL}$ \\
\hline 3 & & & 5 & 76 & $7.5 \mu \mathrm{g} / \mathrm{ml}$ \\
\hline 4 & & & 6 & 73 & $6.0 \mu \mathrm{g} / \mathrm{mL}$ \\
\hline 5 & & $\mathrm{H}$ & 7 & 66 & $4.0 \mu \mathrm{g} / \mathrm{mL}$ \\
\hline 6 & & $\mathrm{H}$ & 9 & 72 & $5.5 \mu \mathrm{g} / \mathrm{mL}$ \\
\hline 7 & & $\mathrm{H}$ & 9 & 76 & $5.0 \mu \mathrm{g} / \mathrm{ml}$ \\
\hline 8 & & $\mathrm{H}$ & 10 & 77 & $1.0 \mu \mathrm{g} / \mathrm{ml}$ \\
\hline
\end{tabular}

from the $\beta$-glucosidase treated prunioside $A$, the present study is the first report that the ester derivatives of prunioside A may be the potential therapeutic agents for some inflammatory diseases.

Acknowledgment. This work was supported by the Medicinal Resources Research Center of Wonkwang University and partially by Wonkwang University in 2002.

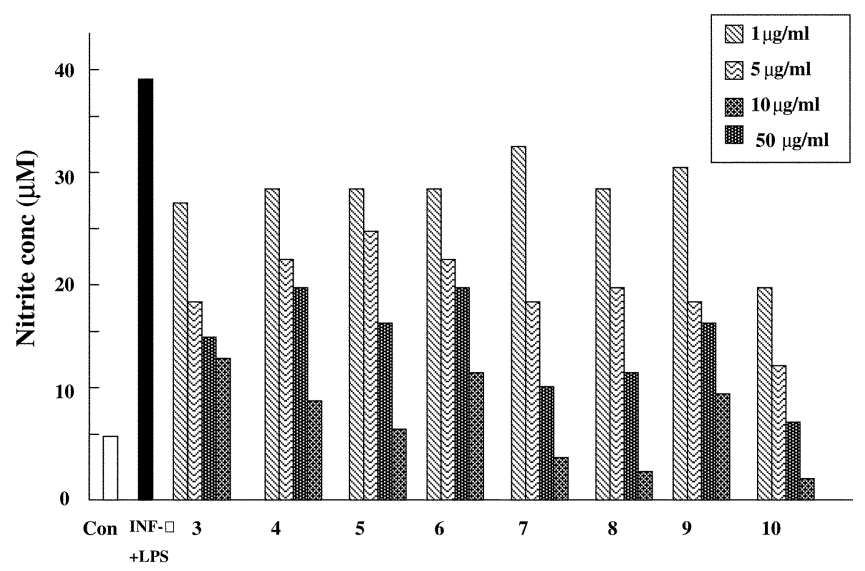

Figure 1. Fillects of compounds 3-10 on NO production in I.PSslimulated RAW 264.7 cells. Cells were stimulated for $24 \mathrm{~h}$ with $\mathrm{l}$ $\mu \mathrm{g} / \mathrm{mL}$ of LPS in the presence or absence of test compounds a1 indicated concentrations.

\section{References}

1. Nathat C. FASEB J. 1992. 6.305I.

2. Moncad. S.: Palmer. R. M, 1.: Iliggs. F. A. Phamerol. Rev 1991. 43. 109.

3. Kilboum. R. G.: Gross. S. S.: Jubran. A.: Adams. I.: Griflith. O. W.: Levi. R. Proc Natl Acod Sol LSA 1990. 87.3629.

4. Lalenti. A.: Ianaro. A.: Moncada. S.: DiRosa. M. Lur .J Hhametcol 1992.211 .177

5. MeCartney-Francis. N.: Allen, J. B.: Mizel. D. F.: Albina J. F.: Xie, Q. W. Nathuus. C. l:. Wall. S. M. J. Exp. Med. 1993. /78. 749 .

6. So. H. S.: l'arh. R.: Oh. H. M.: Pae. H. O.: Lee. J. H.: Chai. K. Y.: Chung. S. Y.: Chung. H. T. f. Fthmophamacol. 1999. 68. 209.

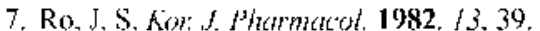

8. Woo. M. I1.: I.ee I: IL: Chung, S. O.: Kim. C. W. Nor J. Pharmacol 1996. 27.389.

9. Oh. H.: Oh. G. S.: Seo. W. G.: I'ate H. O.: Chai. K. Y.: Kwon. I. O.: Lee. Y. H.: Chung. H. T.: Lee. H. S. J. Nat. Prod 2001. ot. 942.

10. Oh, I1.: Shin, 11,: Oh, G. S.: Pat, II, O.: Chai, K. Y.: Chung. H. T.: Lee. II. S. Phytochem. 2003. 64. 1113. 